\title{
The freeness of Ish arrangements
}

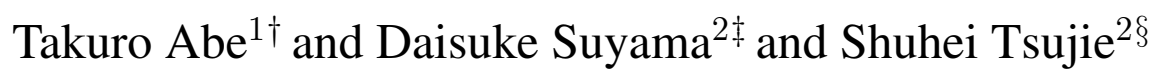 \\ ${ }^{1}$ Department of Mechanical Engineering and Science, Kyoto University, Kyoto 606-8501, Japan. \\ ${ }^{2}$ Department of Mathematics, Hokkaido University, Sapporo, Hokkaido 060-0810, Japan.
}

\begin{abstract}
The Ish arrangement was introduced by Armstrong to give a new interpretation of the $q, t$-Catalan numbers of Garsia and Haiman. Armstrong and Rhoades showed that there are some striking similarities between the Shi arrangement and the Ish arrangement and posed some problems. One of them is whether the Ish arrangement is a free arrangement or not. In this paper, we verify that the Ish arrangement is supersolvable and hence free. Moreover, we give a necessary and sufficient condition for the deleted Ish arrangement to be free.
\end{abstract}

Résumé. L'arrangement Ish a été introduit par Armstrong pour donner une nouvelle interprétation des nombres $q, t$ Catalan de Garsia et Haiman. Armstrong et Rhoades ont montré qu'il y avait des ressemblances frappantes entre l'arrangement Shi et l'arrangement Ish et ont posé des conjectures. L'une d'elles est de savoir si l'arrangement Ish est un arrangement libre ou pas. Dans cet article, nous vérifions que l'arrangement Ish est supersoluble et donc libre. De plus, on donne une condition nécessaire et suffisante pour que l'arrangement Ish réduit soit libre.

Keywords: Hyperplane arrangement, Ish arrangement, Shi arrangement, Coxeter arrangement, Supersolvable arrangement

\section{Introduction}

Let $\mathbb{K}$ be a field of characteristic 0 and $\left\{x_{1}, \ldots, x_{\ell}\right\}$ a basis for the dual space $\left(\mathbb{K}^{\ell}\right)^{*}$ of the $\ell$-dimensional vector space $\mathbb{K}^{\ell}$. The Coxeter arrangement $\operatorname{Cox}(\ell)$ of type $A_{\ell-1}$ (also called the braid arrangement) is

$$
\operatorname{Cox}(\ell):=\left\{\left\{x_{i}-x_{j}=0\right\} \mid 1 \leq i<j \leq \ell\right\},
$$

where $\{x=k\}\left(x \in\left(\mathbb{K}^{\ell}\right)^{*}, k \in \mathbb{K}\right)$ is the affine hyperplane $\left\{v \in \mathbb{K}^{\ell} \mid x(v)=k\right\}$. Then the Shi arrangement $\operatorname{Shi}(\ell)$ and the Ish arrangement $\operatorname{Ish}(\ell)$ are defined by

$$
\begin{aligned}
& \operatorname{Shi}(\ell):=\operatorname{Cox}(\ell) \cup\left\{\left\{x_{i}-x_{j}=1\right\} \mid 1 \leq i<j \leq \ell\right\}, \\
& \operatorname{Ish}(\ell):=\operatorname{Cox}(\ell) \cup\left\{\left\{x_{1}-x_{j}=i\right\} \mid 1 \leq i<j \leq \ell\right\} .
\end{aligned}
$$

The Shi arrangement originally defined over $\mathbb{R}$ was introduced by J.Y. Shi [Shi (1986)] in the study of the Kazhdan-Lusztig representation theory of the affine Weyl groups. The Ish arrangement also originally

\footnotetext{
†Email: abe.takuro.4cekyoto-u.ac.jp.

¥Email: dsuyama@math. sci.hokudai.ac.jp.

$\S$ Email: tsujie@math.sci.hokudai.ac.jp.
} 
defined over $\mathbb{R}$ was introduced by Armstrong in [Armstrong (2013)]. He gave a new interpretation of the $q, t$-Catalan numbers of Garsia and Haiman by using these two arrangements. Armstrong and Rhoades showed that there are some striking similarities between the Shi arrangement and the Ish arrangement in [Armstrong (2013); Armstrong and Rhoades (2012)].

Let $\mathcal{A}$ be an arrangement in $\mathbb{K}^{\ell}$. Let $L(\mathcal{A})$ be the set of nonempty intersections of hyperplanes in $\mathcal{A}$, which is partially ordered by the reverse inclusion of subspaces. Define the Möbius function $\mu: L(\mathcal{A}) \rightarrow$ $\mathbb{Z}$ as follows:

$$
\begin{gathered}
\mu\left(\mathbb{K}^{\ell}\right)=1, \\
\mu(X)=-\sum_{\mathbb{K}^{\ell} \leq Y<X} \mu(Y)\left(X \neq \mathbb{K}^{\ell}\right) .
\end{gathered}
$$

Then the characteristic polynomial $\chi(\mathcal{A}, t) \in \mathbb{Z}[t]$ of $\mathcal{A}$ is defined by

$$
\chi(\mathcal{A}, t)=\sum_{X \in L(\mathcal{A})} \mu(X) t^{\operatorname{dim} X} .
$$

The following theorem is one of the similarities pointed out by Armstrong.

Theorem 1.1 ([Armstrong (2013); Headley (1997)]) The characteristic polynomial of the Shi arrangement and the Ish arrangement are given by

$$
\chi(\operatorname{Shi}(\ell), t)=\chi(\operatorname{Ish}(\ell), t)=t(t-\ell)^{\ell-1} .
$$

Let $\left\{x_{1}, \ldots, x_{\ell}, z\right\}$ be a basis for $V^{*}$ of $V:=\mathbb{K}^{\ell+1}$. Then, as in [(Orlik and Terao, 1992, Definition 1.15)], we have the cone $\mathbf{c}(\operatorname{Ish}(\ell))$ over the Ish arrangement which is a central arrangement (Namely, an arrangement whose hyperplanes pass through the origin) in $V$ defined by

$$
Q(\mathbf{c}(\operatorname{Ish}(\ell)))=z \prod_{1 \leq i<j \leq \ell}\left(x_{i}-x_{j}\right)\left(x_{1}-x_{j}-i z\right)=0 .
$$

Let $S$ be the symmetric algebra of the dual space $V^{*} . S$ can be identified with the polynomial ring $\mathbb{K}\left[x_{1}, \ldots, x_{\ell}, z\right]$. Let $\operatorname{Der}(S)$ be the module of derivations of $S$

$$
\operatorname{Der}(S):=\{\theta: S \rightarrow S \mid \theta \text { is } \mathbb{K} \text {-linear, } \theta(f g)=f \theta(g)+\theta(f) g \text { for any } f, g \in S\} .
$$

Then, for a central arrangement $\mathcal{A}$ in $V$, the module of logarithmic derivations $D(\mathcal{A})$ of $\mathcal{A}$ is defined to be

$$
\begin{aligned}
D(\mathcal{A}) & :=\{\theta \in \operatorname{Der}(S) \mid \theta(Q(\mathcal{A})) \in Q(\mathcal{A}) S\} \\
& =\left\{\theta \in \operatorname{Der}(S) \mid \theta\left(\alpha_{H}\right) \in \alpha_{H} S \text { for any } H \in \mathcal{A}\right\},
\end{aligned}
$$

where $Q(\mathcal{A})$ is the defining polynomial of $\mathcal{A}$ and $\alpha_{H}$ is a linear form such that $\operatorname{ker}\left(\alpha_{H}\right)=H$. We say that $\mathcal{A}$ is free if $D(\mathcal{A})$ is a free $S$-module. Then $D(\mathcal{A})$ has a homogeneous basis $\left\{\theta_{0}, \ldots, \theta_{\ell}\right\}$ and the tuple of degrees $\exp \mathcal{A}=\left(\operatorname{deg} \theta_{0}, \ldots, \operatorname{deg} \theta_{\ell}\right)$ is called the exponents of $\mathcal{A}$.

The main purpose of this paper is to settle a problem of whether the Ish arrangements are free or not, which was posed by Armstrong and Rhoades in [(Armstrong and Rhoades. 2012, p. 1527, (3))]. We define a new class of arrangements which is a generalization of the Ish arrangements and will characterize free arrangements in this class. 
Definition 1.2 Let $N=\left(N_{2}, N_{3}, \ldots, N_{\ell}\right)$ be a tuple of finite subsets $N_{j}$ in $\mathbb{K}$. Define the $N$-Ish arrangement $\operatorname{Ish}(N)$ by

$$
\operatorname{Ish}(N):=\left\{\left\{x_{1}-x_{j}=a\right\} \mid 2 \leq j \leq \ell, a \in N_{j}\right\}
$$

$$
\cup\left\{\left\{x_{i}-x_{j}=0\right\} \mid 2 \leq i<j \leq \ell\right\} .
$$

We say that $N$ is a nest if there exists a permutation $w$ of $\{2, \ldots, \ell\}$ such that

$$
N_{w(2)} \subseteq N_{w(3)} \subseteq \cdots \subseteq N_{w(\ell)} .
$$

In particular, when $N_{j}=\{0,1, \ldots, j-1\}$ for each $j$, the $N$-Ish arrangement $\operatorname{Ish}(N)$ is the Ish arrangement $\operatorname{Ish}(\ell)$. We denote the cone over the $N$-Ish arrangement $\mathbf{c}(\operatorname{Ish}(N))$ by $\mathcal{I}=\mathcal{I}_{N}$. The defining polynomial of $\mathcal{I}$ can be expressed as

$$
Q(\mathcal{I})=z\left(\prod_{j=2}^{\ell} \prod_{a \in N_{j}}\left(x_{1}-x_{j}-a z\right)\right)\left(\prod_{2 \leq i<j \leq \ell}\left(x_{i}-x_{j}\right)\right) .
$$

Our main results are as follows:

Theorem 1.3 The following four conditions are equivalent:

(1) $N$ is a nest.

(2) $\mathcal{I}_{N}$ is supersolvable.

(3) $\mathcal{I}_{N}$ is inductively free.

(4) $\mathcal{I}_{N}$ is free.

The definitions of supersolvable and inductively free arrangements will be mentioned in Section 2. Note that the implications $(2) \Rightarrow(3) \Rightarrow(4)$ are general properties for arrangements [Orlik and Terao (1992)]. This theorem asserts that there are no differences among these properties for $N$-Ish arrangements.

Theorem 1.4 Let $N=\left(N_{2}, N_{3}, \ldots, N_{j}\right)$ with $N_{2} \subseteq N_{3} \subseteq \cdots \subseteq N_{j}$. Define homogeneous derivations $\theta_{0}, \theta_{1}, \ldots, \theta_{\ell}$ by

$$
\begin{aligned}
\theta_{0} & :=\sum_{i=1}^{\ell} \frac{\partial}{\partial x_{i}}, \quad \theta_{1}=\sum_{i=1}^{\ell} x_{i} \frac{\partial}{\partial x_{i}}+z \frac{\partial}{\partial z}, \\
\theta_{k} & :=\sum_{s=2}^{k}\left(\prod_{a \in N_{k}}\left(x_{1}-x_{s}-a z\right) \prod_{t=k+1}^{\ell}\left(x_{s}-x_{t}\right)\right) \frac{\partial}{\partial x_{s}}(2 \leq k \leq \ell) .
\end{aligned}
$$

Then $\theta_{0}, \theta_{1}, \ldots, \theta_{\ell}$ form a basis for $D\left(\mathcal{I}_{N}\right)$. In particular, the exponents are given by

$$
\exp \mathcal{I}_{N}=\left(0,1,\left|N_{2}\right|+\ell-2,\left|N_{3}\right|+\ell-3, \ldots,\left|N_{\ell}\right|\right)
$$

where $\left|N_{j}\right|$ denotes the cardinality of $N_{j}$. 
Corollary 1.5 The cone over the Ish arrangement $\mathbf{c}(\operatorname{Ish}(\ell))$ is free with exponents $\exp (\mathbf{c}(\operatorname{Ish}(\ell)))=$ $(0,1, \underbrace{\ell, \ell, \ldots, \ell}_{(\ell-1) \text { times }})$. Moreover the homogeneous derivations

$$
\begin{aligned}
& \theta_{0}=\sum_{i=1}^{\ell} \frac{\partial}{\partial x_{i}}, \quad \theta_{1}=\sum_{i=1}^{\ell} x_{i} \frac{\partial}{\partial x_{i}}+z \frac{\partial}{\partial z}, \\
& \theta_{k}=\sum_{s=2}^{k}\left(\prod_{i=0}^{k-1}\left(x_{1}-x_{s}-i z\right) \prod_{t=k+1}^{\ell}\left(x_{s}-x_{t}\right)\right) \frac{\partial}{\partial x_{s}}(2 \leq k \leq \ell)
\end{aligned}
$$

form a basis for $D(\mathbf{c}(\operatorname{Ish}(\ell)))$.

If an arrangement $\mathcal{A}$ is a free arrangement, then the characteristic polynomial of $\mathcal{A}$ can be expressed by using its exponents:

Theorem 1.6 ([Terao $(1981)])$ If an arrangement $\mathcal{A}$ is free with exponents $\left(d_{1}, \ldots, d_{\ell}\right)$, then the characteristic polynomial of $\mathcal{A}$ splits as

$$
\chi(\mathcal{A}, t)=\prod_{i=1}^{\ell}\left(t-d_{i}\right) .
$$

Since we have the relation between the characteristic polynomials of $\mathcal{A}$ and $\mathbf{c} \mathcal{A}$

$$
\chi(\mathbf{c} \mathcal{A}, t)=(t-1) \chi(\mathcal{A}, t),
$$

we obtain a new proof of Theorem 1.1 from Corollary 1.5 and Theorem 1.6

The complement $M(\mathcal{A}):=\mathbb{K}^{\ell} \backslash \cup_{H \in \mathcal{A}} H$ of a supersolvable arrangement $\mathcal{A}$ has very interesting properties: If $\mathbb{K}=\mathbb{C}$, the complement $M(\mathcal{A})$ is fiber type [Terao (1986)]. In particular, $M(\mathcal{A})$ is a $K(\pi, 1)$ space, i.e., the homotopy groups $\pi_{i}(M(\mathcal{A}))=0$ for $i \geq 2$. When $\mathbb{K}=\mathbb{R}$, the complement $M(\mathcal{A})$ is a disjoint union of chambers. For chambers $C, C^{\prime}$, define $d\left(C, C^{\prime}\right)$ by the number of hyperplanes in $\mathcal{A}$ separating $C$ from $C^{\prime}$. Björner, Edelman, and Ziegler [Björner et al. (1990)] gave the wall-crossing formula as follows: There exists a base chamber $B$ of $\mathcal{A}$ such that

$$
\sum_{C \in \operatorname{Ch}(\mathcal{A})} t^{d(B, C)}=\prod_{i=1}^{\ell}\left(1+t+\cdots+t^{d_{i}}\right),
$$

where $\left(d_{1}, \ldots, d_{\ell}\right)$ is the exponents of $\mathcal{A}$ and $\operatorname{Ch}(\mathcal{A})$ denotes the set of all chambers of $\mathcal{A}$. Therefore, we derive the following corollary from our main theorems 1.3 and 1.4

Corollary 1.7 Let $N=\left(N_{2}, N_{3}, \ldots, N_{j}\right)$ with $N_{2} \subseteq N_{3} \subseteq \cdots \subseteq N_{j}$.

(1) If $\mathbb{K}=\mathbb{C}$, then the complement $M\left(\mathcal{I}_{N}\right)$ of the cone over the $N$-Ish arrangement $\mathcal{I}_{N}$ is $K(\pi, 1)$.

(2) If $\mathbb{K}=\mathbb{R}$, then there exists a base chamber $B \in \operatorname{Ch}\left(\mathcal{I}_{N}\right)$ such that

$$
\sum_{C \in \operatorname{Ch}\left(\mathcal{I}_{N}\right)} t^{d(B, C)}=t \prod_{i=2}^{\ell}\left(1+t+\cdots+t^{\left|N_{i}\right|+\ell-i}\right) .
$$


The organization of this paper is as follows. In Section 2, we review the theory of supersolvable arrangements and prove Theorem 1.3. In Section 3, we verify Theorem 1.4 applying Saito's criterion. In Section 4, we recall the deleted arrangement $\operatorname{Shi}(G)$ and $\operatorname{Ish}(G)$ defined by Armstrong and Rhoades in [Armstrong and Rhoades (2012)] and prove that $\operatorname{Shi}(G)$ and $\operatorname{Ish}(G)$ share the freeness.

\section{Supersolvability and freeness of $\mathcal{I}$}

For an arrangement $\mathcal{A}$, let $L(\mathcal{A})$ be the set of nonempty intersections of hyperplanes in $\mathcal{A}$. If $\mathcal{A}$ is central, then $L(\mathcal{A})$ is a geometric lattice with the order by reverse inclusion: $X \leq Y \Leftrightarrow Y \subseteq X$. In the rest of this section, "arrangement" means "central arrangement". The rank of an arrangement $\mathcal{A}$, denoted by $\operatorname{rank}(\mathcal{A})$, is the codimension of $\cap_{H \in \mathcal{A}} H$. We say that $\mathcal{A}$ is essential if $\operatorname{rank}(\mathcal{A})$ is equal to the dimension of the ambient space of $\mathcal{A}$.

An arrangement $\mathcal{A}$ is supersolvable if the intersection lattice $L(\mathcal{A})$ is supersolvable as defined by Stanley [Stanley $[(1972)]$. The following lemma is widely known.

Lemma 2.1 ([Terao $(1986)])$ An arrangement $\mathcal{A}$ is supersolvable if and only if there exists a filtration

$$
\mathcal{A}=\mathcal{A}_{\ell} \supseteq \mathcal{A}_{\ell-1} \supseteq \cdots \supseteq \mathcal{A}_{1}
$$

such that

(1) $\operatorname{rank}\left(\mathcal{A}_{i}\right)=i(i=1,2, \ldots, \ell)$.

(2) For any $H, H^{\prime} \in \mathcal{A}_{i}$ with $H \neq H^{\prime}$, there exists some $H^{\prime \prime} \in \mathcal{A}_{i-1}$ such that $H \cap H^{\prime} \subseteq H^{\prime \prime}$.

Let $\mathcal{A}$ be an arrangement. For a hyperplane $H \in \mathcal{A}$, define arrangements

$$
\mathcal{A}^{\prime}:=\mathcal{A} \backslash\{H\} \text { and } \mathcal{A}^{\prime \prime}:=\left\{H^{\prime} \cap H \mid H^{\prime} \in \mathcal{A}^{\prime}\right\} .
$$

The tuple $\left(\mathcal{A}, \mathcal{A}^{\prime}, \mathcal{A}^{\prime \prime}\right)$ is called the triple of arrangements with respect to $H$. For a triple $\left(\mathcal{A}, \mathcal{A}^{\prime}, \mathcal{A}^{\prime \prime}\right)$, the Addition Theorem [Terao (1980a b)] asserts that if $\mathcal{A}^{\prime}$ and $\mathcal{A}^{\prime \prime}$ are free and $\exp \mathcal{A}^{\prime \prime} \subset \exp \mathcal{A}^{\prime}$, then $\mathcal{A}$ is free.

Definition 2.2 Define the inductive freeness by the following:

(1) The empty arrangement is inductively free.

(2) $\mathcal{A}$ is inductively free if there exists $H \in \mathcal{A}$ such that $\mathcal{A}^{\prime}$ and $\mathcal{A}^{\prime \prime}$ are inductively free and $\exp \mathcal{A}^{\prime \prime} \subset$ $\exp \mathcal{A}^{\prime}$.

Thanks to the Addition Theorem, the inductive freeness implies the freeness. Moreover, it is also known that the the supersolvability implies the inductive freeness (see [(Orlik and Terao, 1992, Theorem 4.58)] for example).

We will use the following lemma which is a part of the Addition-Deletion Theorem:

Lemma 2.3 ([( Orlik and Terao, 1992. Theorem 4.46)]) Let $\left(\mathcal{A}, \mathcal{A}^{\prime}, \mathcal{A}^{\prime \prime}\right)$ be a triple. Suppose that $\mathcal{A}$ is an essential arrangement of rank 3 and that arrangements $\mathcal{A}^{\prime}$ and $\mathcal{A}^{\prime \prime}$ are free with $\exp \left(\mathcal{A}^{\prime}\right)=(1, a, b)$ and $\exp \left(\mathcal{A}^{\prime \prime}\right)=(1, c)$. If $c \notin\{a, b\}$ then $\mathcal{A}$ is not free. 
We are now prepared to prove Theorem 1.3

Proof of Theorem 1.3: (1) $\Rightarrow(2)$ Without loss of generality, we may assume that $N_{2} \supseteq N_{3} \supseteq \cdots \supseteq N_{\ell}$. For each $i \in\{1,2, \ldots, \ell\}$, define $X_{i} \in L(\mathcal{I})$ by

$$
X_{i}:=\left\{z=x_{1}-x_{2}=\cdots=x_{1}-x_{i}=0\right\} .
$$

Then the rank of the localization $\mathcal{I}_{i}:=\mathcal{I}_{X_{i}}=\{H \in \mathcal{I} \mid H \supseteq X\}$ is equal to $i$ and we have

$$
\begin{aligned}
& \mathcal{I}_{i}=\left\{\left\{x_{1}-x_{j}=a z\right\} \mid 1<j \leq i \text { and } a \in N_{j}\right\} \\
& \cup\left\{\left\{x_{j}-x_{k}=0\right\} \mid 2 \leq j<k \leq i\right\} \cup\{\{z=0\}\}
\end{aligned}
$$

Hence there exists a filtration

$$
\mathcal{I}=\mathcal{I}_{\ell} \supseteq \mathcal{I}_{\ell-1} \supseteq \cdots \supseteq \mathcal{I}_{1}
$$

By Lemma 2.1, we have only to verify that for any $H, H^{\prime} \in \mathcal{I}_{i}$ with $H \neq H^{\prime}$ there exists some $H^{\prime \prime} \in \mathcal{I}_{i-1}$ such that $H \cap H^{\prime} \subseteq H^{\prime \prime}$ for each $i \in\{2, \ldots, \ell\}$. We may assume that both $H$ and $H^{\prime}$ do not belong to $\mathcal{I}_{i-1}$. Then $H$ and $H^{\prime}$ belong to

$$
\mathcal{I}_{i} \backslash \mathcal{I}_{i-1}=\left\{\left\{x_{1}-x_{i}=a z\right\} \mid a \in N_{i}\right\} \cup\left\{\left\{x_{j}-x_{i}=0\right\} \mid 2 \leq j<i\right\} .
$$

First, let $a$ and $b$ be distinct elements in $N_{i}$. Suppose that $H=\left\{x_{1}-x_{i}=a z\right\}$ and $H^{\prime}=\left\{x_{1}-x_{i}=b z\right\}$. Then $H \cap H^{\prime} \subseteq\{z=0\} \in \mathcal{I}_{i-1}$. Second, let $j$ and $k$ be distinct integers in $\{2, \ldots, i-1\}$. Assume that $H=\left\{x_{j}-x_{i}=0\right\}$ and $H^{\prime}=\left\{x_{k}-x_{i}=0\right\}$. Then $H \cap H^{\prime} \subseteq\left\{x_{j}-x_{k}=0\right\} \in \mathcal{I}_{i-1}$. Finally, let $H=\left\{x_{1}-x_{i}=a z\right\}$ and $H^{\prime}=\left\{x_{j}-x_{i}=0\right\}$ with $a \in N_{i}$ and $2 \leq j<i$. Then $H \cap H^{\prime} \subseteq\left\{x_{1}-x_{j}=a z\right\} \in \mathcal{I}_{i-1}$ by the assumption $a \in N_{i} \subseteq N_{j}$. Thus the cone over the $N$-Ish arrangement $\mathcal{I}$ is supersolvable.

$(2) \Rightarrow(3) \Rightarrow(4)$ We have nothing to prove as mentioned before.

$(4) \Rightarrow(1)$ When $\ell=2$, the tuple $N=\left(N_{2}\right)$ is a nest. For $\ell \geq 3$, we will prove that if $N$ is not a nest then $\mathcal{I}$ is not free by induction on $\ell$. First, let $\ell=3$. Then we have $N=\left(N_{2}, N_{3}\right)$. Let $H \in \mathcal{I}$ be the hyperplane $\left\{x_{2}-x_{3}=0\right\}$ and $\left(\mathcal{I}, \mathcal{I}^{\prime}, \mathcal{I}^{\prime \prime}\right)$ the triple with respect to $H$. One can verify easily that the homogeneous derivations

$$
\sum_{i=1}^{3} x_{i} \frac{\partial}{\partial x_{i}}+z \frac{\partial}{\partial z}, \quad \prod_{a \in N_{2}}\left(x_{1}-x_{2}-a z\right) \frac{\partial}{\partial x_{2}}, \quad \prod_{a \in N_{3}}\left(x_{1}-x_{3}-a z\right) \frac{\partial}{\partial x_{3}}
$$

form a basis for $D\left(\mathcal{I}^{\prime}\right)$ (with the non-essential derivation $\sum_{i=1}^{3} \frac{\partial}{\partial x_{i}}+\frac{\partial}{\partial z}$ ). Hence the arrangement $\mathcal{I}^{\prime}$ is free with exponents $\left(1,\left|N_{2}\right|,\left|N_{3}\right|\right)$. The arrangement $\mathcal{I}^{\prime \prime}$ is also free with exponents $\left(1,\left|N_{2} \cup N_{3}\right|\right)$ since $\operatorname{rank}\left(\mathcal{I}^{\prime \prime}\right)=2$ and $\left|\mathcal{I}^{\prime \prime}\right|=1+\left|N_{2} \cup N_{3}\right|$. By the assumption, $N$ is not a nest, i.e., $N_{2} \nsubseteq N_{3}$ and $N_{2} \nsupseteq N_{3}$, hence we have that $\left|N_{2} \cup N_{3}\right|$ is strictly larger than both of $\left|N_{2}\right|$ and $\left|N_{3}\right|$. Therefore, by Lemma 2.3, we have concluded that $\mathcal{I}$ is not free.

Now suppose that $\ell>3$. Since $N$ is not a nest, there exist integers $i, j$ such that $N_{i} \nsubseteq N_{j}$ and $N_{i} \nsupseteq N_{j}$. Define $X \in L(\mathcal{I})$ by

$$
X:=\left\{z=x_{1}-x_{i}=x_{1}-x_{j}=0\right\} .
$$


Then we have

$$
\mathcal{I}_{X}=\left\{\left\{x_{1}-x_{k}=a z\right\} \mid k \in\{i, j\} \text { and } a \in N_{k}\right\} \cup\left\{\left\{x_{i}-x_{j}=0\right\},\{z=0\}\right\} .
$$

Hence $\mathcal{I}_{X}$ is equivalent to $\mathbf{c}\left(\operatorname{Ish}\left(N_{i}, N_{j}\right)\right)$ discussed in the above paragraph. Therefore the localization $\mathcal{I}_{X}$ is not free, neither is $\mathcal{I}$.

\section{A basis for $D(\mathcal{I})$}

In this section, we will prove Theorem 1.4 First, we verify that $\theta_{0}, \theta_{1}, \ldots, \theta_{\ell}$ belong to $D(\mathcal{I})$.

Lemma 3.1 Let $N=\left(N_{2}, N_{3}, \ldots, N_{j}\right)$ with $N_{2} \subseteq N_{3} \subseteq \cdots \subseteq N_{j}$. Then

$$
\begin{aligned}
& \theta_{0}=\sum_{i=1}^{\ell} \frac{\partial}{\partial x_{i}}, \quad \theta_{1}=\left(\sum_{i=1}^{\ell} x_{i} \frac{\partial}{\partial x_{i}}\right)+z \frac{\partial}{\partial z}, \\
& \theta_{k}=\sum_{s=2}^{k}\left(\prod_{a \in N_{k}}\left(x_{1}-x_{s}-a z\right) \prod_{t=k+1}^{\ell}\left(x_{s}-x_{t}\right)\right) \frac{\partial}{\partial x_{s}}(2 \leq k \leq \ell)
\end{aligned}
$$

belong to $D(\mathcal{I})$.

Proof: Since $\theta_{0}\left(\alpha_{H}\right)=0$ for any $H \in \mathcal{I}$, it belongs to $D(\mathcal{I})$. The Euler derivation $\theta_{1}$ belongs to $D(\mathcal{A})$ for any central arrangement $\mathcal{A}$, thus $\theta_{1} \in D(\mathcal{I})$. We will show that $\theta_{k} \in D(\mathcal{I})$ for $2 \leq k \leq \ell$. It is obvious that $\theta_{k}(z)=0 \in z S$.

Let $2 \leq i<j \leq \ell$.

Case 1. If $i<j \leq k$, then

$$
\begin{aligned}
\theta_{k}\left(x_{i}-x_{j}\right)= & \left(\prod_{a \in N_{k}}\left(x_{1}-x_{i}-a z\right) \prod_{t=k+1}^{\ell}\left(x_{i}-x_{t}\right)\right) \\
& -\left(\prod_{a \in N_{k}}\left(x_{1}-x_{j}-a z\right) \prod_{t=k+1}^{\ell}\left(x_{j}-x_{t}\right)\right) \\
\equiv & -\left(\prod_{a \in N_{k}}\left(x_{1}-x_{i}-a z\right) \prod_{t=k+1}^{\ell}\left(x_{i}-x_{t}\right)\right) \\
& =0
\end{aligned}
$$

thus $\theta_{k}\left(x_{i}-x_{j}\right) \in\left(x_{i}-x_{j}\right) S$. 
Case 2. If $i \leq k<j$, then

$$
\theta_{k}\left(x_{i}-x_{j}\right)=\prod_{a \in N_{k}}\left(x_{1}-x_{i}-a z\right) \prod_{t=k+1}^{\ell}\left(x_{i}-x_{t}\right) \in\left(x_{i}-x_{j}\right) S .
$$

Case 3. If $k<i<j$, then

$$
\theta_{k}\left(x_{i}-x_{j}\right)=0 \in\left(x_{i}-x_{j}\right) S
$$

Hence $\theta_{k}\left(x_{i}-x_{j}\right) \in\left(x_{i}-x_{j}\right) S$ for $2 \leq i<j \leq \ell$.

Let $2 \leq j \leq \ell$ and $b \in N_{j}$.

Case 1. If $j \leq k$, then $b \in N_{j} \subseteq N_{k}$, thus

$$
\begin{aligned}
\theta_{k}\left(x_{1}-x_{j}-b z\right) & =\prod_{a \in N_{k}}\left(x_{1}-x_{j}-a z\right) \prod_{t=k+1}^{\ell}\left(x_{j}-x_{t}\right) \\
& \in\left(x_{1}-x_{j}-b z\right) S .
\end{aligned}
$$

Case 2. If $k<j$, then

$$
\theta_{k}\left(x_{1}-x_{j}-b z\right)=0 \in\left(x_{1}-x_{j}-b z\right) S
$$

Hence $\theta_{k}\left(x_{1}-x_{j}-b z\right) \in\left(x_{1}-x_{j}-b z\right) S$ for $2 \leq j \leq \ell$ and $b \in N_{j}$. Therefore we obtain that $\theta_{k} \in D(\mathcal{I})$.

\section{Proof of Theorem 1.4}

First, note that if $s=1, k \geq 2$ then

$$
\theta_{k}\left(x_{s}\right)=\theta_{k}\left(x_{1}\right)=0
$$

and if $2 \leq k<s$ then

$$
\theta_{k}\left(x_{s}\right)=0
$$

Thus the determinant of the coefficient matrix of $\theta_{0}, \theta_{1}, \ldots, \theta_{\ell}$ can be calculated as follows: 


$$
\begin{aligned}
\left|\begin{array}{cccc}
\theta_{0}\left(x_{1}\right) & \theta_{1}\left(x_{1}\right) & \cdots & \theta_{\ell}\left(x_{1}\right) \\
\vdots & \vdots & \cdots & \vdots \\
\theta_{0}\left(x_{\ell}\right) & \theta_{1}\left(x_{\ell}\right) & \cdots & \theta_{\ell}\left(x_{\ell}\right) \\
\theta_{0}(z) & \theta_{1}(z) & \cdots & \theta_{\ell}(z)
\end{array}\right| & =\left|\begin{array}{ccccc}
1 & x_{1} & 0 & \cdots & 0 \\
1 & x_{2} & \theta_{2}\left(x_{2}\right) & \cdots & \theta_{\ell}\left(x_{2}\right) \\
\vdots & \vdots & 0 & \ddots & \vdots \\
1 & x_{\ell} & \vdots & \ddots & \theta_{\ell}\left(x_{\ell}\right) \\
0 & z & 0 & \cdots & 0
\end{array}\right| \\
& \doteq z\left|\begin{array}{ccccc}
1 & 0 & 0 & \cdots & 0 \\
1 & \theta_{2}\left(x_{2}\right) & \theta_{3}\left(x_{2}\right) & \cdots & \theta_{\ell}\left(x_{2}\right) \\
1 & & \theta_{3}\left(x_{3}\right) & \cdots & \theta_{\ell}\left(x_{3}\right) \\
\vdots & 0 & \ddots & \vdots \\
1 & 0 & \theta_{\ell}\left(x_{\ell}\right)
\end{array}\right| \\
& =z \prod_{k=2}^{\ell} \theta_{k}\left(x_{k}\right) \\
& =z \prod_{k=2}^{\ell}\left(\prod_{a \in N_{k}}\left(x_{1}-x_{k}-a z\right) \prod_{t=k+1}^{\ell}\left(x_{k}-x_{t}\right)\right) \\
& =z\left(\prod_{k=2}^{\ell} \prod_{a \in N_{k}}\left(x_{1}-x_{k}-a z\right)\right)\left(\prod_{k=2}^{\ell} \prod_{t=k+1}^{\ell}\left(x_{k}-x_{t}\right)\right) \\
& =z\left(\prod_{k=2}^{\ell} \prod_{a \in N_{k}}\left(x_{1}-x_{k}-a z\right)\right)\left(\prod_{2 \leq k<t \leq \ell}\left(x_{k}-x_{t}\right)\right) \\
& =Q(\mathcal{I}),
\end{aligned}
$$

where $\doteq$ denotes that they are equal up to a nonzero constant multiple. Combining this calculation and Lemma 3.1. we can apply Saito's criterion [Saito (1980)] and see that $\theta_{0}, \theta_{1}, \ldots, \theta_{\ell}$ form a basis for $D(\mathcal{I})$.

\section{Freeness of the deleted Ish arrangements}

Let $K_{\ell}$ be the complete graph on $\ell$ vertices. We can regard $K_{\ell}$ as the set of directed edges $(i, j)(i<j)$, namely $K_{\ell}=\{(i, j) \mid 1 \leq i<j \leq \ell\}$. For a subgraph $G \subseteq K_{\ell}$, Armstrong and Rhoades [Armstrong and Rhoades (2012)] defined the deleted arrangements $\operatorname{Shi}(G)$ and $\operatorname{Ish}(G)$ and showed that they share many properties. In particular, it was proven that $\operatorname{Shi}(G)$ and $\operatorname{Ish}(G)$ have the same characteristic polynomials by their explicit expressions. The deleted Shi and Ish arrangements are defined by

$$
\begin{aligned}
\operatorname{Shi}(G) & :=\operatorname{Cox}(\ell) \cup\left\{\left\{x_{i}-x_{j}=1\right\} \mid(i, j) \in G\right\} \subseteq \operatorname{Shi}(\ell), \\
\operatorname{Ish}(G) & :=\operatorname{Cox}(\ell) \cup\left\{\left\{x_{1}-x_{j}=i\right\} \mid(i, j) \in G\right\} \subseteq \operatorname{Ish}(\ell) .
\end{aligned}
$$

Athanasiadis gave a necessary and sufficient condition for the freeness of $\mathbf{c}(\operatorname{Shi}(G))$. 
Theorem 4.1 (Athanasiadis (1998) Theorem 4.1) Let $G \subseteq K_{\ell}$ be a subgraph. The cone over the deleted Shi arrangement $\mathbf{c}(\operatorname{Shi}(G))$ is free if and only if there exists a permutation $w$ of $\{1, \ldots, \ell\}$ such that $w^{-1} G$ is contained in $K_{\ell}$, i.e., $(i, j) \in w^{-1} G$ implies $i<j$, and has the following property:

$$
\text { If } 1 \leq i<j<k \leq \ell \text { and }(i, j) \in w^{-1} G \text { then }(i, k) \in w^{-1} G .
$$

In this section, we will prove that the property of $G$ in the Theorem 4.1 is also a necessary and sufficient condition for the freeness of $\mathbf{c}(\operatorname{Ish}(G))$ by making use of the terminology of the $N$-Ish arrangements. The problem of whether the cone of the deleted Ish arrangement $\mathbf{c}(\operatorname{Ish}(G))$ is free or not is posed by Armstrong and Rhoades in [( Armstrong and Rhoades, 2012, p. 1517)] together with the problem for $\mathbf{c}(\operatorname{Ish}(\ell))$.

For a subgraph $G \subseteq K_{\ell}$, define a tuple of sets $N_{G}=\left(N_{2}, \ldots, N_{\ell}\right)$ by

$$
N_{j}:=\{0\} \cup\{i \mid(i, j) \in G\} \subseteq\{0,1, \ldots, j-1\} .
$$

It is easy to show that $\operatorname{Ish}\left(N_{G}\right)=\operatorname{Ish}(G)$.

Theorem 4.2 Let $G \subseteq K_{\ell}$ be a subgraph. Then the following are equivalent:

(1) $\mathbf{c}(\operatorname{Ish}(G))$ is free.

(2) $N_{G}$ is a nest.

(3) G has the property in Theorem 4.1.

(4) For any $j, k \in\{2, \ldots, \ell\}$, either of the following two conditions holds:

(i) If $(i, j) \in G$ then $(i, k) \in G$ for any $i \leq \min \{j, k\}$.

(ii) If $(i, k) \in G$ then $(i, j) \in G$ for any $i \leq \min \{j, k\}$.

Proof: $(1) \Leftrightarrow(2)$ It is obvious from Theorem 1.3

$(2) \Rightarrow(3)$ Assume that $N_{G}$ is a nest. Then there exists a permutation $w$ of $\{1, \ldots, \ell\}$ with $w(1)=1$ such that

$$
N_{w(2)} \subseteq N_{w(3)} \subseteq \cdots \subseteq N_{w(\ell)} .
$$

Now, we will prove that $w^{-1} G \subseteq K_{\ell}$ i.e., $(i, j) \in w^{-1} G$ implies $i<j$. For any $(i, j) \in w^{-1} G$, we have $(w(i), w(j)) \in G$. Hence $w(i) \in N_{w(j)}$. Then $N_{w(j)} \nsubseteq N_{w(i)}$ since $w(i) \notin N_{w(i)}$. Since $N_{G}$ is a nest, we have $N_{w(i)} \subseteq N_{w(j)}$. Therefore $i<j$, namely $(i, j) \in K_{\ell}$. Thus we have showed that $w^{-1} G \subseteq K_{\ell}$.

Suppose that $1 \leq i<j<k \leq \ell$. Then we have a chain of implications:

$$
\begin{aligned}
(i, j) \in w^{-1} G & \Rightarrow(w(i), w(j)) \in G \Rightarrow w(i) \in N_{w(j)} \\
& \Rightarrow w(i) \in N_{w(k)} \Rightarrow(w(i), w(k)) \in G \Rightarrow(i, k) \in w^{-1} G .
\end{aligned}
$$

This proves that $G$ satisfies the second condition.

$(3) \Rightarrow(4)$ Fix elements $j, k \in\{2, \ldots, \ell\}$ and assume that $w^{-1}(j)<w^{-1}(k)$. For any $(i, j) \in G$, we have that $\left(w^{-1}(i), w^{-1}(j)\right) \in w^{-1} G$. Since $w^{-1} G \subseteq K_{\ell}$, we have $w^{-1}(i)<w^{-1}(j)$. Then the 
second property of (3) implies $\left(w^{-1}(i), w^{-1}(k)\right) \in w^{-1} G$, i.e., $(i, k) \in G$. Hence (i) holds. Similarly, if $w^{-1}(j)>w^{-1}(k)$ then (ii) holds.

(4) $\Rightarrow(2)$ For any $j, k \in\{2, \ldots, \ell\}$, it is clear that (i) holds if and only if $N_{j} \subseteq N_{k}$ and (ii) holds if and only if $N_{k} \subseteq N_{j}$. Therefore every element in $N_{G}$ is comparable. Thus $N_{G}$ is a nest.

Combining Theorem 4.1 and Theorem 4.2 , we can prove that the following corollary:

Corollary 4.3 The deleted arrangements $\operatorname{Shi}(G)$ and $\operatorname{Ish}(G)$ share the freeness.

\section{Acknowledgements}

The authors thank the referees for careful reading and many useful comments on this article.

\section{References}

D. Armstrong. Hyperplane arrangements and diagonal harmonics. J. Comb., 4(2):157-190, 2013. ISSN 2156-3527.

D. Armstrong and B. Rhoades. The Shi arrangement and the Ish arrangement. Trans. Amer. Math. Soc., 364(3):1509-1528, 2012. ISSN 0002-9947.

C. A. Athanasiadis. On free deformations of the braid arrangement. European J. Combin., 19(1):7-18, 1998. ISSN 0195-6698.

A. Björner, P. H. Edelman, and G. M. Ziegler. Hyperplane arrangements with a lattice of regions. Discrete Comput. Geom., 5(3):263-288, 1990. ISSN 0179-5376.

P. Headley. On a family of hyperplane arrangements related to the affine Weyl groups. J. Algebraic Combin., 6(4):331-338, 1997. ISSN 0925-9899.

P. Orlik and H. Terao. Arrangements of hyperplanes, volume 300 of Grundlehren der Mathematischen Wissenschaften [Fundamental Principles of Mathematical Sciences]. Springer-Verlag, Berlin, 1992. ISBN 3-540-55259-6.

K. Saito. Theory of logarithmic differential forms and logarithmic vector fields. J. Fac. Sci. Univ. Tokyo Sect. IA Math., 27(2):265-291, 1980. ISSN 0040-8980.

J. Y. Shi. The Kazhdan-Lusztig cells in certain affine Weyl groups, volume 1179 of Lecture Notes in Mathematics. Springer-Verlag, Berlin, 1986. ISBN 3-540-16439-1.

R. P. Stanley. Supersolvable lattices. Algebra Universalis, 2:197-217, 1972. ISSN 0002-5240.

H. Terao. Arrangements of hyperplanes and their freeness. I. J. Fac. Sci. Univ. Tokyo Sect. IA Math., 27 (2):293-312, 1980a. ISSN 0040-8980.

H. Terao. Arrangements of hyperplanes and their freeness. II. The Coxeter equality. J. Fac. Sci. Univ. Tokyo Sect. IA Math., 27(2):313-320, 1980b. ISSN 0040-8980.

H. Terao. Generalized exponents of a free arrangement of hyperplanes and Shepherd-Todd-Brieskorn formula. Invent. Math., 63(1):159-179, 1981. ISSN 0020-9910. 
H. Terao. Modular elements of lattices and topological fibration. Adv. in Math., 62(2):135-154, 1986. ISSN 0001-8708. 